\title{
Detection and analysis of antibiotic resistance variability among Staphylococcus aureus isolates from animal and human sources
}

\author{
Sandeep K. Sharma ${ }^{1 *}$, Rahul Yadav ${ }^{1}$, Sharat C. Mehta ${ }^{2}$, and Anil K. Kataria ${ }^{1}$ \\ ${ }^{1}$ Department of Veterinary Microbiology and Biotechnology, College of Veterinary and Animal Science, Bikaner, \\ Rajasthan University of Veterinary and Animal Sciences, Bikaner, (Rajasthan), India \\ ${ }^{2}$ Animal Genetics and Breeding Unit, National Research Centre on Camel, Indian Council of Agricultural Research \\ (ICAR) Bikaner, India
}

SHARMA, S. K., R. YADAV, S. C. MEHTA, A. K. KATARIA: Detection and analysis of antibiotic resistance variability among Staphylococcus aureus isolates from animal and human sources. Vet. arhiv 90, 493-508, 2020.

\section{ABSTRACT}

In consideration of the importance of Staphylococcus aureus regarding its contribution to antibiotic resistance, the present study was designed to find variability among $S$. aureus isolates in relation to their multidrug resistance patterns. A total of 157 species-specific $23 \mathrm{~S}$ rRNA based confirmed $S$. aureus isolates from various clinical and nonclinical animal sources (cattle, buffalo, goat, sheep, dog, camel, pig and horse), human and pieces of meat from butcher shops were included in the present study. Overall more than $95 \%$ isolates were recorded resistant to ampicillin and penicillin-G, while approximately $100 \%$ isolates were sensitive to chloramphenicol, meropenem and nitrofurantoin. The isolates from different sources showed highly significant $(\mathrm{P} \leq 0.01)$ variation in their resistance patterns for 39 antibiotics, significant variation $(\mathrm{P} \leq 0.05)$ for levofloxacin and nitrofurantoin, and no significant variation $(\mathrm{P}>0.05)$ for clindamycin. In Bonferroni correction, human isolates were significantly variable with a $\mathrm{P}<0.0001$ probability level of variance in relation to other pieces of meat and animal origin sources for most of the antibiotics. Human isolates had the highest (0.40) MAR index. A highly significant difference was observed in the antibiogram pattern between different sources of $S$. aureus, which may indicate the pattern and frequency of use of various antibiotics in humans and animals.

Key words: Staphylococcus aureus; antibiotic resistance; animal; human; DMRT analysis

\section{Introduction}

Staphylococcus aureus is a notorious member of the Staphylococcaceae family associated with various clinical and sub clinical infections. In humans, it may also cause serious infections, particularly in persons debilitated by chronic illness, traumatic injury, burns and immunosuppression. These infections include pneumonia, deep abscesses, osteomyelitis, endocarditis, phlebitis, boils, furuncles, styes, impetigo, toxic shock syndrome and meningitis, and are often associated worldwide with hospitalized patients rather than healthy individuals in the community (CARTER et al., 1990). This organism is also known to cause a variety of suppurative infections, septicemia and

\footnotetext{
*Corresponding author:

Assist. Prof. Sandeep Kumar Sharma, MVSc, PhD, Department of Veterinary Microbiology and Biotechnology, PGIVER, Jaipur, (RAJUVAS, Bikaner), India, Phone: +91 9414775 879; E-mail: drsharmask01@hotmail.com
} 
toxinoses in domestic animals and/or birds, such as: mastitis, udder impetigo, endometritis, pyoderma, cystitis, dermatitis, botryomycosis of mammary glands, arthritis, scirrhous cord, bumble foot and tick pyaemia (NABER, 2009).

Although $S$. aureus is naturally susceptible to many antibiotics, it is acquiring antimicrobial resistance for multifactorial reasons that include the excessive widespread and inappropriate use of antimicrobials, the extensive use of these agents as growth enhancers in animal feed, and, with the increase in regional and international travel, the relative ease with which antimicrobial-resistant bacteria cross geographical barriers (LOWY, 2003). Staphylococci have developed many efficient mechanisms to neutralize new antibiotics, namely: enzymatic inactivation of the antibiotic, alteration of the target with decreased affinity for the antibiotic, trapping of the antibiotic, and efflux pumps. Complex genetic arrays have been acquired by $S$. aureus through horizontal gene transfer, while resistance to other antibiotics, including some of the most recent ones (e.g., fluoroquinolones, linezolid and daptomycin), has developed through spontaneous chromosomal and plasmid mutations and antibiotic positive selection (LOWY, 2003; PANTOSTI et al., 2007).

In the course of resistance development, $S$. aureus showed resistance towards penicillin by the production of a specific enzyme called penicillinase ( $\beta$-lactamase), often governed by genes located in plasmids. This is an extracellular enzyme, hydrolyzing the $\beta$-lactam ring of $\beta$-lactam antibiotics (penicillin) (LOWY, 2003). Methicillin resistant $S$. aureus (MRSA) is an important resistant phenotype of $S$. aureus which has acquired resistance through PBP2a (penicillin binding protein 2a) through blocking the proteins responsible for the construction and maintenance of the bacterial cell wall. The PBP2a protein is encoded by the mecA gene, which is the hallmark of MRSA. As opposed to the penicillinase gene, mecA does not reside on plasmids but on the chromosome, embedded in a large mobile genetic element called staphylococcal chromosome cassette mec or SCCmec. The presence of PBP2a means MRSA is not only resistant to methicillin, but also to all $\beta$-lactam antibiotics, including synthetic penicillins, cephalosporins and carbapenems (PANTOSTI and VENDITTI, 2009).

Vancomycin was identified as the first line of drug for treatment of MRSA, thus unfortunately use of vancomycin dramatically increased, not only for $S$. aureus infections but also for other infections, which resulted in the emergence of vancomycin resistance among bacterial populations (LOOMBA et al., 2010). Establishment of MRSA and the emergence of VRSA are of great concern because these are not only resistant to methicillin, but also to vancomycin, monobactams and cephalosporins, through the production of ESBL (Extendedspectrum beta-lactamases). Antibiotic resistance, the overuse of antibiotics, increased healthcare costs and sepsis-related deaths give rise to the need for exploration of the phenotypic and genotypic variations of resistance among $S$. aureus strains obtained from human and animal infections.

Considering the importance of $S$. aureus as the cause of various ailments in humans and animals, and variations in the strains in relation to antimicrobial resistance, the present study was undertaken with the objective of discovering the antibiotic resistance patterns of $S$. aureus from clinical and non-clinical sources of human and animal origin, and their statistical analysis, to ascertain the variable associations between isolates, according to their source of origin.

\section{Material and methods}

Bacterial strain. In the present investigation, 157 genotypically confirmed (species specific 23S rRNA sequence based based) $S$. aureus isolates were examined, of which 35 isolates were obtained from clinical infections of humans, 20 from pieces of meat from butcher's shops, three from horse skin wounds, two from pig nasal cavities, eight from camel skin wounds, six from dog skin infections, six from clinical infections of sheep, 21 from mastitic buffalo milk, 28 from mastitic goats' milk and 28 isolates were obtained from mastitic cows' milk samples.

Antibiotics susceptibility testing. The sensitivity test was conducted using the method of BAUER et al. (1966), against 42 antibiotics of different generations (Table 1), belonging to various groups. 
The isolates were inoculated into sterile $5 \mathrm{~mL}$ Mueller-Hinton Broth tubes, and incubated for 18 hours at $37{ }^{\circ} \mathrm{C}$. The opacity was adjusted to 0.5 McFarland opacity standard, and inoculums were well spread over the Mueller-Hinton agar surface, with the help of a sterilized spreader. The plates were allowed to dry for 10 minutes at $37^{\circ} \mathrm{C}$, and then antibiotic discs (Table 1, Hi Media, Mumbai) were carefully placed on the surface with enough space around each disc for diffusion of the antibiotic. The plates were incubated for 24 hours at $37^{\circ} \mathrm{C}$, and the diameter of the zone of inhibition of growth around each disc was measured in millimeters. After measurement of the inhibition zone, interpretation of resistant, sensitive and intermediates was made using the breakpoints defined by The Clinical \& Laboratory Standards Institute (CLSI, 2016).

Multiple antibiotic resistance index (MAR) value. All multidrug resistant isolates were evaluated for their Multiple Antibiotic Resistance (MAR) index. In assessment of MDR isolates, this index was calculated as per the method given by KRUMPERMAN (1983).

$M A R$ Index of single isolate $=a / b$, where $a$ represents the number of antibiotics to which the isolate was resistant and $b$ represents the number of antibiotics to which the isolate was exposed.

Group MAR index value $=a /\left(b^{*} c\right)$, where $a$ is the aggregate antibiotic resistance score of all isolates in the group, $b$ is the number of antibiotics to which the isolate was exposed, and $c$ is the total number of isolates in the group

Statistical analysis. For comparative analysis and to find variability between the antibiotic resistance patterns of $S$. aureus isolates of different origins, the diameter of the zone of inhibition of various antibiotics was recorded separately for each isolate. Then the mean diameter of the zone of inhibition of the isolates (ANOVA), the Duncan multiple range test (DMRT) analysis, Bonferroni correction and frequency of resistant, sensitive and intermediates were determined according to the method of CAMPBELL et al. (2007), using Microsoft excel and IBM SPSS Advanced Statistics 20.0 version software.

\section{Results}

In the present investigation, 157 Staphylococcus aureus isolates obtained from animals (cattle, buffalo, goat, sheep, dog, camel, pig and horse), humans and pieces of meat from local butcher's shops were subjected to detection and analysis of antibiotic resistance patterns against 42 antibiotics belonging to different categories and generations. The isolates exhibited significant variations in sensitivity patterns (Table 1). Overall, it was recorded that all $(100 \%)$ isolates were sensitive to meropenem, chloramphenicol (except one human isolate, H28), and nitrofurantoin (except one cattle isolate, C39), more than $85 \%$ isolates were sensitive to nine antibiotics, namely: ampicillin+sulbactum (96.2\%), imipenem (95.5\%), cefalothin (91.7\%), piperacillin+ tazobactam (91.7\%), tobramycin (89.8\%), doxycycline hydrochlorid (89.2\%), ceftazidime + clavulanic acid $(88.5 \%)$, oxacillin $(87.3 \%)$ and polymxin-B (86.0\%). More than $95.0 \%$ isolates were resistant to ampicillin and penicillin-G. Statistical analysis revealed that the Mean Sum of Square (MSS) of the diameter of the inhibition zone $(\mathrm{mm})$ of each antibiotic showed highly significant $(\mathrm{P} \leq 0.01)$ variations between isolates from different sources in their resistance patterns for 39 antibiotics, significant variations $(\mathrm{P} \leq 0.05)$ for levofloxacin and nitrofurantoin, and a non-significant variation $(\mathrm{P}>0.05)$ for clindamycin (Table 2).

In the present study, highly significant $(\mathrm{P} \leq 0.01)$ variations were recorded between $S$. aureus isolates for most of the antibiotics, thus all sources of isolates were further subjected to Duncan's Homogeneous Subsets analysis (DMRT) of Mean \pm SEM values of the diameter of inhibition zone of the antibiotics. In the analysis, a maximum of five subsets were found for five antibiotics, namely: cefaclor, cefixime, cefixime + clavulanic acid, imipenem and ticarcillin, and four subsets were found for 13 antibiotics, namely: ampicillin, ampicillin+ sulbactum, cefoparazone, ceftazidime+clavulanic acid, doxycycline hydrochloride, linezolid, nitrofurantoin, norfloxacin, penicillin-G, piperacillin, piperacillin + tazobactam, tetracycline and ticarcillin + clavulanic acid among $S$. aureus isolates. For the other antibiotics, two or three subsets were found among $S$. aureus isolates. 


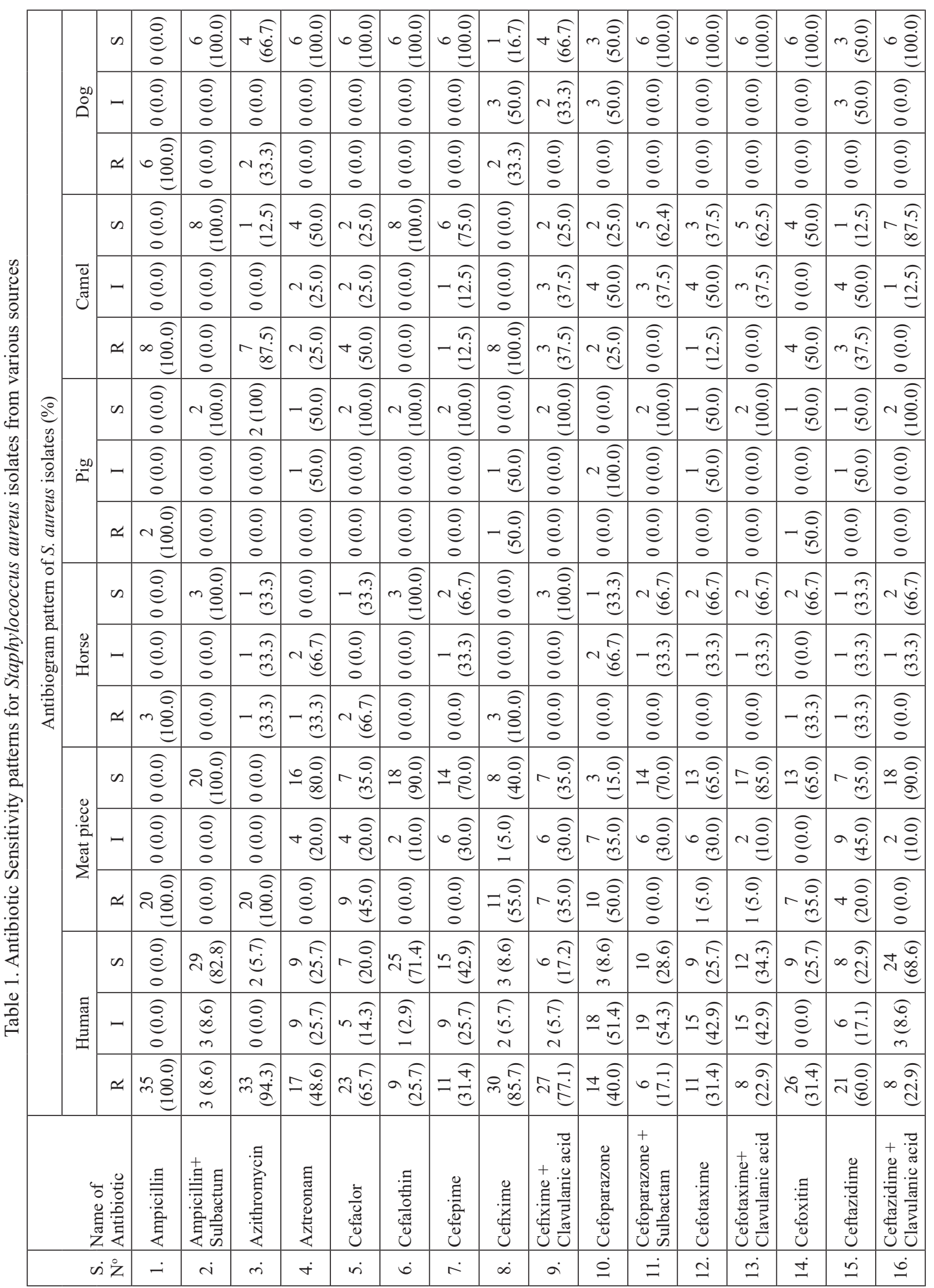




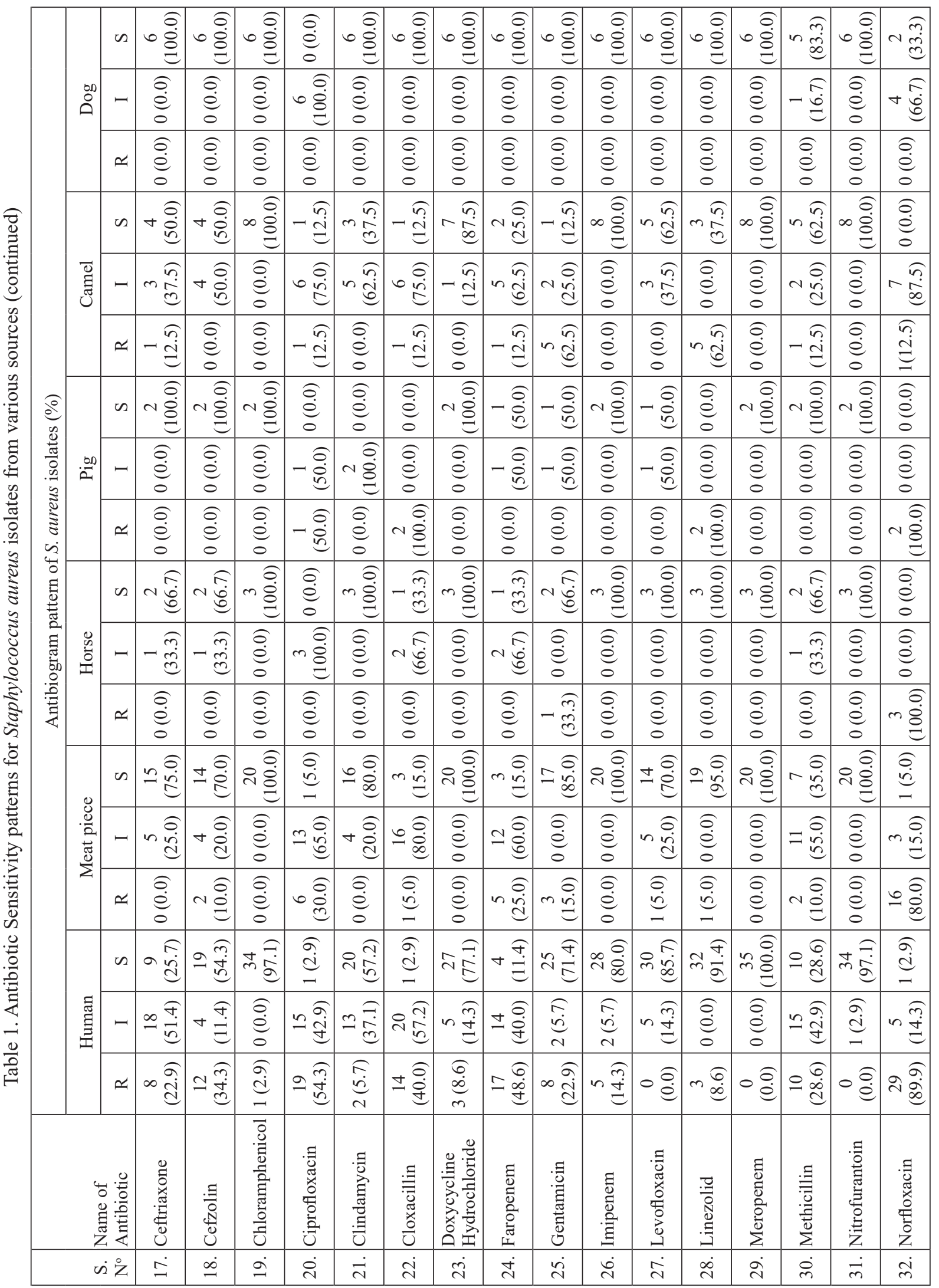




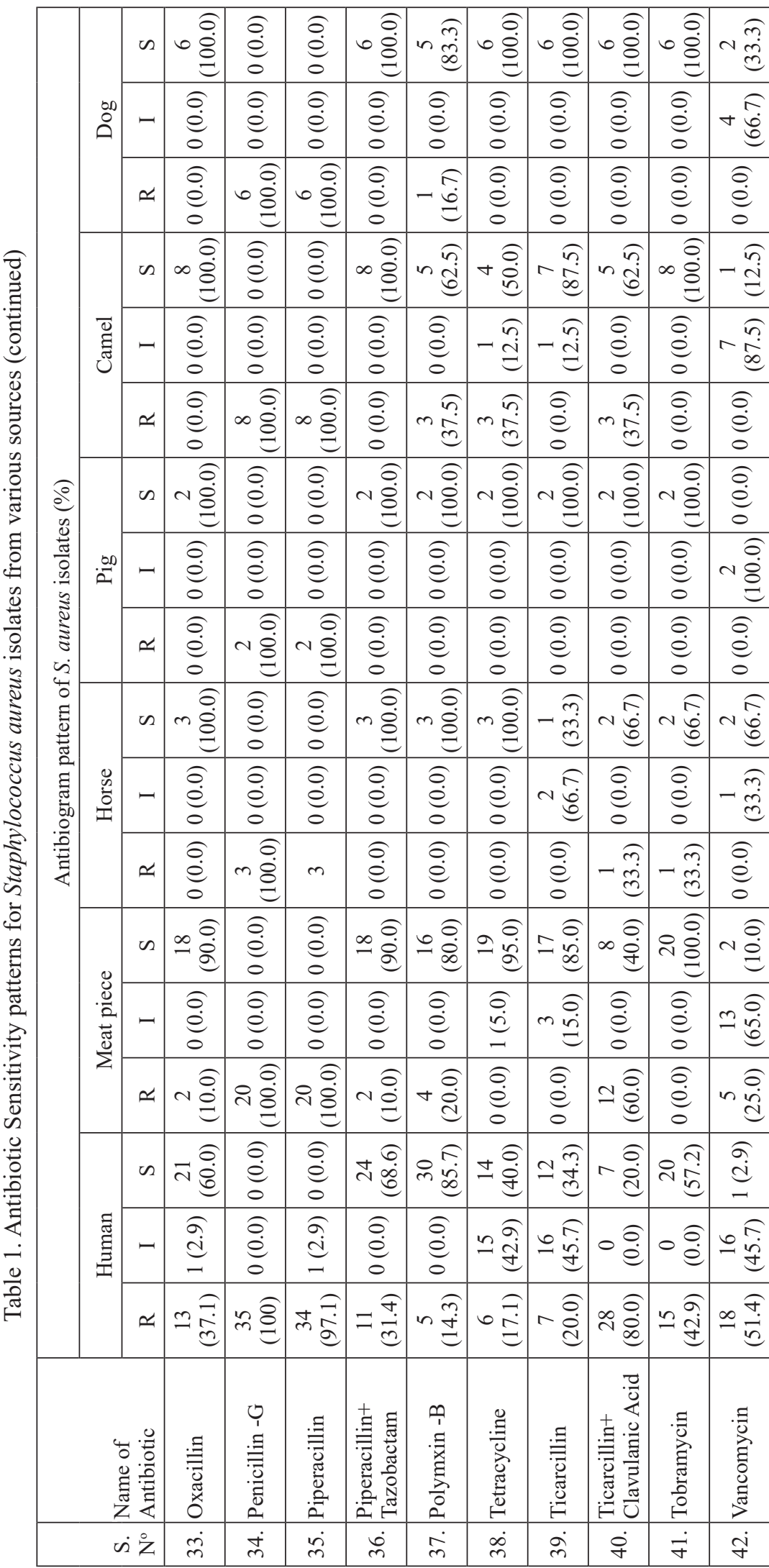

\begin{tabular}{|c|c|c|c|c|c|}
\hline & & Us & $\underset{\substack{n \\
d}}{d}$ & 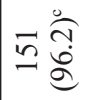 & $\hat{6} \tilde{g}$ \\
\hline & 吾 & - & 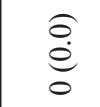 & $\underset{m}{\stackrel{\sigma}{\rightleftarrows}}$ & $\begin{array}{l}\widehat{\sigma} \\
\dot{\infty} \\
\dot{J}\end{array}$ \\
\hline & & $\simeq$ & 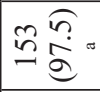 & $m \stackrel{\overbrace{}}{\varrho}$ & 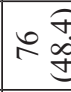 \\
\hline & & $n$ & $\underset{n}{\stackrel{\overbrace{}}{\ominus}}$ & 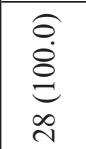 & 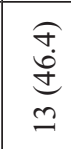 \\
\hline & U & - & $\begin{array}{l}\hat{0} \\
\stackrel{e}{0} \\
0\end{array}$ & 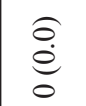 & $\underset{n}{\stackrel{\sigma}{\Sigma}}$ \\
\hline 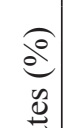 & & $\simeq$ & 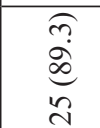 & $\stackrel{0}{\stackrel{0}{e}}$ & $\begin{array}{l}\hat{\sigma} \\
\hat{d} \\
0 \\
0\end{array}$ \\
\hline $\begin{array}{l}\frac{\pi}{0} \\
0 \\
0 \\
0 \\
0 \\
0\end{array}$ & & U & $\stackrel{\substack{n \\
-}}{-}$ & 胥 & $\begin{array}{l}\sigma \\
\dot{i} \\
\hat{\imath} \\
\vec{d}\end{array}$ \\
\hline $\begin{array}{c}\tilde{z} \\
\dot{n} \\
\dot{0}\end{array}$ & ర్ల్ర & - & $\stackrel{\hat{o}}{\stackrel{0}{0}}$ & $\stackrel{0}{\stackrel{0}{0}}$ & $\underset{\forall}{\stackrel{\overbrace{}}{\rightleftarrows}}$ \\
\hline 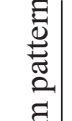 & & $\simeq$ & 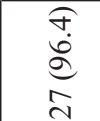 & $\begin{array}{l}\hat{0} \\
\stackrel{e}{0}\end{array}$ & $\underset{m}{\stackrel{\overbrace{}}{g}}$ \\
\hline 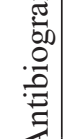 & & U & $\stackrel{0}{\stackrel{\leftrightarrow}{0}}$ & $\underset{\vec{\sigma}}{\stackrel{0}{\dot{\theta}}}$ & 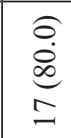 \\
\hline & $\begin{array}{l}\frac{0}{\pi} \\
\stackrel{\mathbb{E}}{\Xi} \\
\stackrel{0}{\Xi}\end{array}$ & - & $\stackrel{\hat{O}}{\stackrel{0}{0}}$ & 官 & 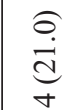 \\
\hline & & $\simeq$ & $\stackrel{\Xi}{\varrho}$ & $\stackrel{\hat{O}}{\stackrel{\leftrightarrow}{0}}$ & $\begin{array}{l}\stackrel{\partial}{e} \\
\stackrel{0}{0}\end{array}$ \\
\hline & & Us & $\stackrel{0}{\stackrel{0}{0}}$ & $\underset{0}{\stackrel{0}{8}}$ & $\underset{0}{\stackrel{0}{8}}$ \\
\hline & $\begin{array}{l}\frac{8}{8} \\
\frac{\tilde{n}}{\sim n}\end{array}$ & - & $\stackrel{0}{\stackrel{0}{0}}$ & $\stackrel{a}{\stackrel{0}{e}}$ & 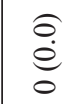 \\
\hline & & $\simeq$ & 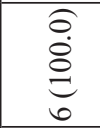 & $\stackrel{0}{\stackrel{0}{0}}$ & $\stackrel{0}{\stackrel{0}{0}}$ \\
\hline & & 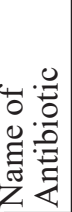 & $\begin{array}{l}: \Xi \\
\stackrel{\Xi}{0} \\
\stackrel{0}{0} \\
\text { 宗 }\end{array}$ & 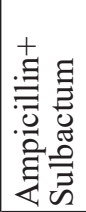 & 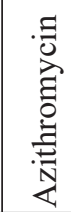 \\
\hline & & $\dot{i n}$ & - & $i$ & $\dot{m}$ \\
\hline
\end{tabular}

Vet. arhiv 90 (5), 493-508, 2020 


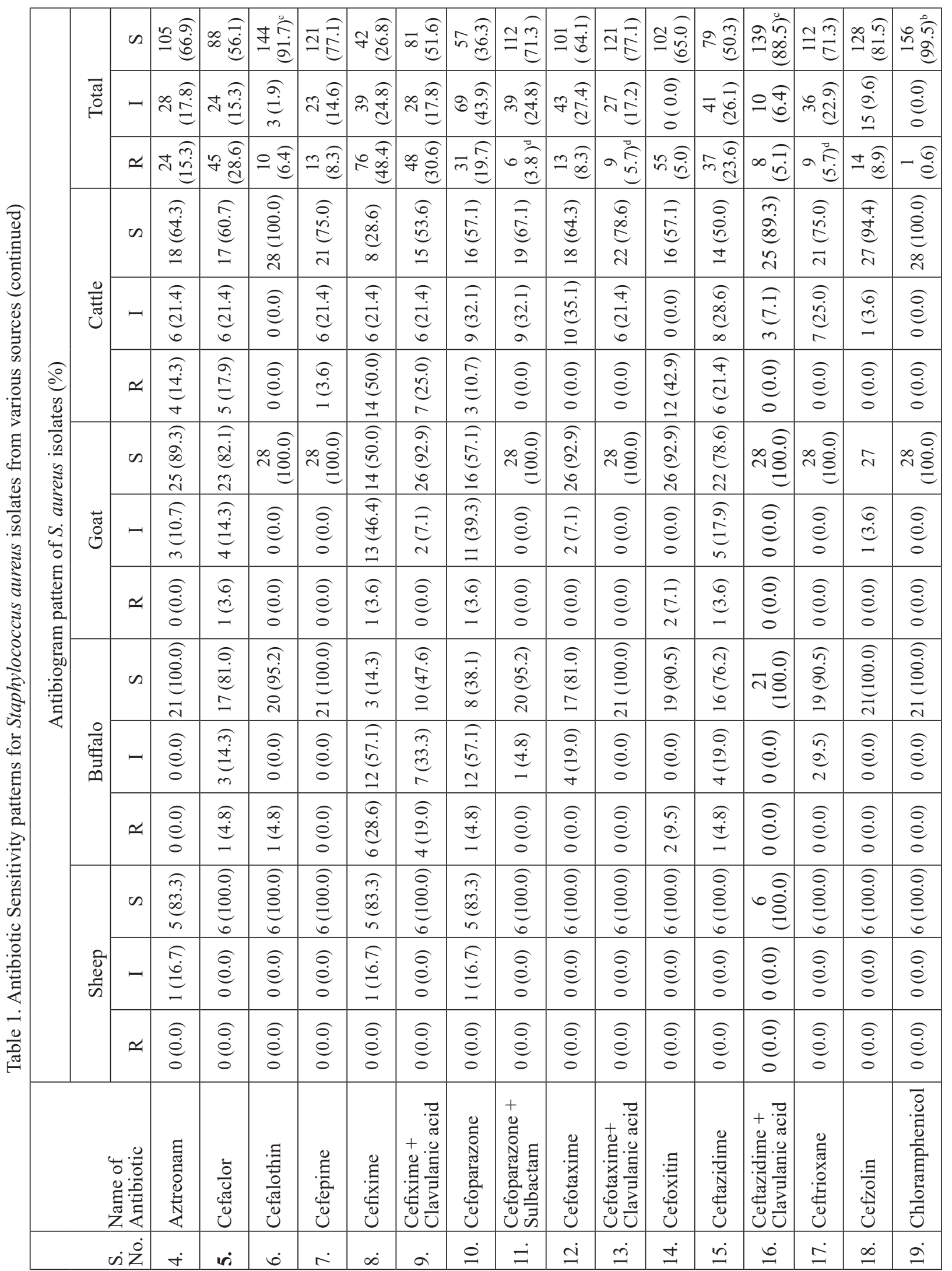




\begin{tabular}{|c|c|c|c|c|c|c|c|c|c|c|c|c|c|c|c|c|}
\hline \multirow{15}{*}{ 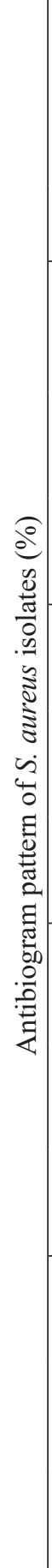 } & \multirow{3}{*}{ 氶 } & 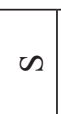 & $\stackrel{\sim}{\stackrel{n}{\infty}}$ & $\stackrel{\overbrace{}}{\approx}$ & 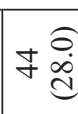 & 守 & $\infty \underset{\overbrace{}}{\overparen{\overbrace{}}}$ & 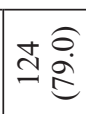 & 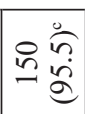 & 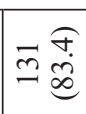 & $m \underset{f}{m}$ & $\hat{n} \stackrel{\varrho}{\dot{\theta}}$. & $\dot{\sigma} \underset{\hat{\sigma}}{\hat{c}}$ & 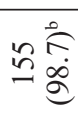 & 字 $\stackrel{0}{\stackrel{0}{0}}$ & 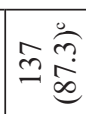 \\
\hline & & - & षे & 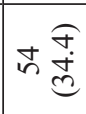 & $\infty \stackrel{\hat{b}}{\stackrel{0}{0}}$ & $\underset{\hat{\sigma}}{\hat{a}}$ & in $\frac{\widehat{\infty}}{-\infty}$ & 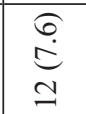 & 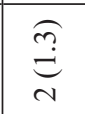 & 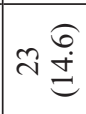 & $\stackrel{\hat{\theta}}{\stackrel{0}{0}}$ & 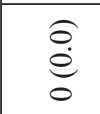 & $\forall \underset{\substack{o \\
\dot{d}}}{\stackrel{d}{d}}$ & $\stackrel{0}{e}$ & 의 & $\underset{m}{\stackrel{\sigma}{\rightleftarrows}}$ \\
\hline & & $\simeq$ & mi & 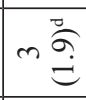 & ป艹 & 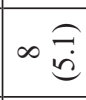 & ๙ 2 & $\vec{\sim} \stackrel{\widehat{f}}{\stackrel{\Xi}{\ominus}}$ & 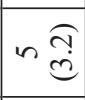 & 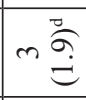 & तָ & $\circ \stackrel{\varrho}{\stackrel{\theta}{e}}$ & $\curvearrowright \widehat{\overparen{I}}$ & $-\stackrel{0}{e}$ & ह) & $=\stackrel{\infty}{\stackrel{0}{\theta}}$ \\
\hline & \multirow{3}{*}{$\underset{\Xi}{\stackrel{\Xi}{\Xi}}$} & Un & 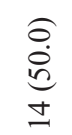 & $\begin{array}{l}\hat{E} \\
\stackrel{8}{0} \\
\text { I }\end{array}$ & $\frac{\underset{d}{d}}{\stackrel{d}{d}}$ & $\begin{array}{l}\vec{\sigma} \\
\dot{i} \\
\stackrel{\vec{\sigma}}{\vec{v}}\end{array}$ & 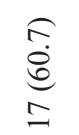 & $\begin{array}{l}\sigma \\
\hat{\sigma} \\
\stackrel{\sigma}{\sigma}\end{array}$ & $\begin{array}{l}\stackrel{0}{0} \\
\stackrel{0}{0} \\
\stackrel{\infty}{\infty}\end{array}$ & 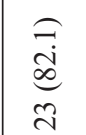 & $\begin{array}{l}\widehat{\sigma} \\
\hat{\sigma} \\
\Omega\end{array}$ & $\underset{\infty}{\stackrel{0}{\stackrel{0}{0}}}$ & $\begin{array}{l}\text { } \\
\underset{0}{0} \\
\stackrel{\infty}{\infty}\end{array}$ & 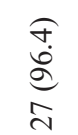 & $\begin{array}{l}\hat{E} \\
\text { id } \\
\text { = }\end{array}$ & 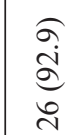 \\
\hline & & $\neg$ & $\begin{array}{l}\hat{\sigma} \\
\stackrel{0}{0} \\
=\end{array}$ & 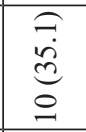 & 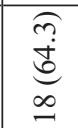 & $\underset{\sim}{\stackrel{\overbrace{}}{(}}$ & 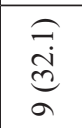 & $\underset{n}{\stackrel{\sigma}{\leftrightarrows}}$ & $\begin{array}{l}\hat{0} \\
\stackrel{0}{0} \\
0\end{array}$ & $\underset{n}{\stackrel{\overbrace{}}{\ominus}}$ & $\begin{array}{l}\hat{0} \\
\stackrel{e}{0} \\
0\end{array}$ & $\underset{0}{\stackrel{0}{e}}$ & $\underset{n}{\stackrel{\sigma}{\Xi}}$ & $\underset{\stackrel{0}{0}}{\stackrel{0}{0}}$ & 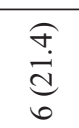 & $\underset{\sim}{\stackrel{\overbrace{}}{\leftrightarrows}}$ \\
\hline & & $\simeq$ & $\underset{m}{\stackrel{\overbrace{}}{g}}$ & $\begin{array}{l}6 \\
\stackrel{0}{0} \\
-\end{array}$ & $\underset{\forall}{\stackrel{\overbrace{}}{\rightleftarrows}}$ & $\underset{n}{\stackrel{\sigma}{\leftrightarrows}}$ & $\underset{\sim}{\stackrel{C}{C}}$ & $\underset{\forall}{\stackrel{\overbrace{}}{\rightleftarrows}}$ & $\begin{array}{l}\hat{\sigma} \\
\stackrel{0}{0} \\
0\end{array}$ & $\underset{\sim}{\stackrel{\overbrace{}}{c}}$ & $\begin{array}{l}\text { तi } \\
\text { d్ } \\
\text { a }\end{array}$ & $\begin{array}{l}\hat{\theta} \\
\dot{e} \\
0\end{array}$ & $\underset{n}{\stackrel{\sigma}{\leftrightarrows}}$ & $\begin{array}{l}6 \\
\stackrel{6}{0} \\
-1\end{array}$ & $\underset{n}{\stackrel{\sigma}{\Xi}}$ & $\begin{array}{l}\hat{0} \\
\stackrel{e}{0} \\
0\end{array}$ \\
\hline & \multirow{3}{*}{ 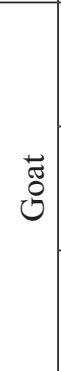 } & an & $\begin{array}{l}\text { ạ } \\
\stackrel{d}{d}\end{array}$ & 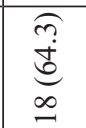 & 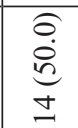 & 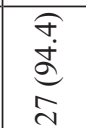 & $\begin{array}{l}\hat{\sigma} \\
\stackrel{\partial}{\infty} \\
\tilde{d}\end{array}$ & 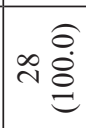 & 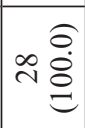 & $\begin{array}{l}\hat{\widehat{a}} \\
\hat{\sigma} \\
\stackrel{d}{0}\end{array}$ & $\begin{array}{l}\hat{\sigma} \\
\stackrel{\partial}{\infty} \\
\tilde{n}\end{array}$ & 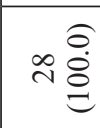 & 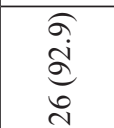 & 兑 & $\begin{array}{l}\hat{\partial} \\
\stackrel{0}{0} \\
\text { In }\end{array}$ & i $\underset{0}{\stackrel{0}{8}}$ \\
\hline & & - & $\begin{array}{l}\widehat{T} \\
\underset{d}{0} \\
\infty \\
-\infty\end{array}$ & 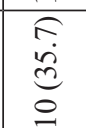 & 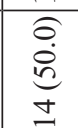 & $\begin{array}{l}\underset{6}{6} \\
\stackrel{-}{c}\end{array}$ & 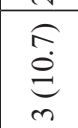 & $\begin{array}{l}\hat{\theta} \\
\stackrel{e}{e} \\
0\end{array}$ & $\begin{array}{l}\hat{0} \\
\dot{e} \\
0\end{array}$ & $\underset{\mathrm{C}}{\stackrel{\overbrace{}}{d}}$ & $\begin{array}{l}\hat{\theta} \\
\dot{e} \\
0\end{array}$ & $\begin{array}{l}\hat{\theta} \\
\stackrel{e}{e} \\
0\end{array}$ & $\underset{\mathrm{N}}{\stackrel{\overbrace{}}{ \pm}}$ & $\begin{array}{l}\hat{\sigma} \\
\stackrel{e}{e} \\
0\end{array}$ & 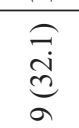 & $\begin{array}{l}\hat{\theta} \\
\stackrel{e}{0}\end{array}$ \\
\hline & & $\simeq$ & $\underset{m}{\stackrel{\overbrace{}}{g}}$ & $\stackrel{0}{\stackrel{\theta}{e}}$ & $\stackrel{0}{\stackrel{\theta}{e}}$ & $\stackrel{0}{\stackrel{0}{e}}$ & $\stackrel{0}{\stackrel{0}{e}}$ & $\stackrel{0}{\stackrel{0}{e}}$ & 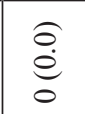 & 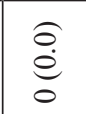 & $\underset{m}{\stackrel{\overbrace{}}{g}}$ & 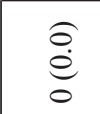 & $\stackrel{0}{\stackrel{\theta}{e}}$ & 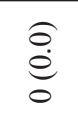 & $\underset{n}{\stackrel{\sigma}{\Xi}}$ & $\stackrel{0}{\dot{e}}$ \\
\hline & \multirow{3}{*}{$\begin{array}{l}\stackrel{0}{\pi} \\
\stackrel{\mathbb{E}}{\leftrightarrows} \\
\stackrel{\oplus}{\Xi}\end{array}$} & U & $\underset{\vec{\sigma}}{\vec{a}}$ & $\begin{array}{l}\hat{\sigma} \\
\underset{\sigma}{\underline{\sigma}} \\
\underline{a}\end{array}$ & $\begin{array}{l}\underset{\sigma}{i} \\
\text { i్ర } \\
=\end{array}$ & $\begin{array}{l}\stackrel{0}{\dot{8}} \\
\stackrel{0}{\Xi} \\
\vec{\sim}\end{array}$ & 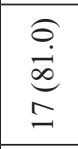 & 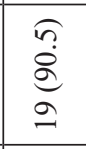 & 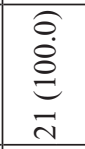 & $\begin{array}{l}\underset{\dot{b}}{\infty} \\
\underline{\infty} \\
\underline{\Xi}\end{array}$ & 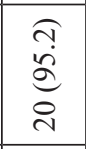 & 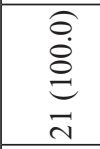 & 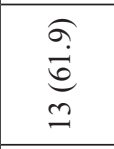 & 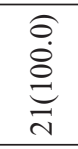 & $\begin{array}{l}0 \\
0\end{array}$ & 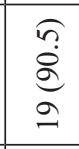 \\
\hline & & $\neg$ & 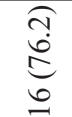 & $\begin{array}{l}\underset{\infty}{\infty} \\
\stackrel{\infty}{\infty} \\
\infty\end{array}$ & $\begin{array}{c}\underset{\infty}{\infty} \\
\stackrel{\infty}{\infty} \\
\infty\end{array}$ & $\underset{\hat{e}}{\stackrel{0}{0}}$ & $\underset{\sigma}{\stackrel{\partial}{\sigma}}$ & $\underset{\sim}{\tilde{a}}$ & 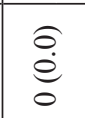 & $\underset{\forall}{\stackrel{\sigma}{a}}$ & 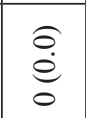 & 官 & 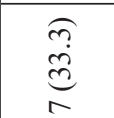 & $\underset{0}{\stackrel{0}{0}}$ & 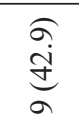 & 官 \\
\hline & & $\simeq$ & $\stackrel{\infty}{\stackrel{\infty}{ \pm}}$ & $\stackrel{0}{\stackrel{0}{e}}$ & $\stackrel{n}{a}$ & 官 & $\stackrel{0}{\stackrel{0}{e}}$ & $\stackrel{a}{\stackrel{0}{0}}$ & $\stackrel{0}{\stackrel{\theta}{e}}$ & $\stackrel{a}{\stackrel{a}{e}}$ & 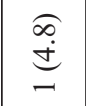 & $\stackrel{0}{\stackrel{0}{e}}$ & $\stackrel{\substack{\infty \\
-}}{-}$ & $\stackrel{0}{\stackrel{0}{e}}$ & $\stackrel{n}{a}$ & $\stackrel{n}{a}$ \\
\hline & \multirow{3}{*}{$\begin{array}{l}\frac{0}{d} \\
\frac{\mathbb{d}}{\omega}\end{array}$} & U & $\underset{\sigma}{\stackrel{\sigma}{\sigma}}$ & 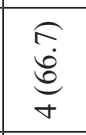 & $\underset{\mathscr{\theta}}{\stackrel{\theta}{\Xi}}$ & $\begin{array}{l}\hat{\sigma} \\
\stackrel{8}{\dot{\theta}} \\
\stackrel{0}{0}\end{array}$ & $\begin{array}{l}\hat{\sigma} \\
\stackrel{8}{8} \\
\stackrel{\theta}{6}\end{array}$ & $\begin{array}{l}\hat{\sigma} \\
\stackrel{8}{8} \\
\stackrel{\theta}{0}\end{array}$ & $\begin{array}{l}\hat{\sigma} \\
\stackrel{8}{\Xi} \\
\stackrel{\theta}{\sigma}\end{array}$ & $\begin{array}{l}\hat{\sigma} \\
\stackrel{8}{\dot{\theta}} \\
\stackrel{0}{0}\end{array}$ & $\begin{array}{l}\hat{\sigma} \\
\stackrel{8}{\dot{\theta}} \\
\stackrel{0}{0}\end{array}$ & $\begin{array}{l}\stackrel{0}{0} \\
\stackrel{8}{\stackrel{8}{0}}\end{array}$ & $\begin{array}{l}\hat{\sigma} \\
\stackrel{8}{\dot{\theta}} \\
\stackrel{0}{0}\end{array}$ & $\begin{array}{l}\hat{\sigma} \\
\stackrel{8}{8} \\
\stackrel{\theta}{\sigma}\end{array}$ & $\begin{array}{l}\hat{0} \\
\stackrel{8}{0} \\
m \\
m\end{array}$ & $\begin{array}{l}\hat{\sigma} \\
\stackrel{8}{\Xi} \\
\stackrel{\theta}{\sigma}\end{array}$ \\
\hline & & - & 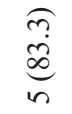 & $\begin{array}{l}\hat{n} \\
\stackrel{\infty}{c} \\
\sim\end{array}$ & $\begin{array}{l}\hat{n} \\
\underset{\infty}{\infty} \\
n\end{array}$ & $\underset{0}{\stackrel{0}{e}}$ & $\stackrel{\hat{O}}{\stackrel{0}{0}}$ & 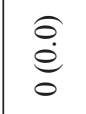 & $\stackrel{\hat{O}}{\stackrel{0}{0}}$ & 官 & 官 & 官 & 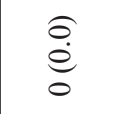 & $\underset{0}{\stackrel{0}{e}}$ & $\begin{array}{l}\text { ô } \\
\stackrel{8}{\circ} \\
\stackrel{n}{n}\end{array}$ & $\underset{0}{\stackrel{\partial}{e}}$ \\
\hline & & $\simeq$ & $\begin{array}{l}\hat{\theta} \\
\stackrel{e}{0} \\
0\end{array}$ & $\underset{0}{\stackrel{0}{0}}$ & $\underset{0}{\stackrel{0}{0}}$ & $\underset{0}{\stackrel{0}{0}}$ & $\begin{array}{l}\hat{\theta} \\
\stackrel{e}{0} \\
0\end{array}$ & $\begin{array}{l}\hat{\theta} \\
\stackrel{e}{0} \\
0\end{array}$ & $\begin{array}{l}\hat{\theta} \\
\stackrel{e}{e} \\
0\end{array}$ & $\underset{0}{\stackrel{0}{e}}$ & $\begin{array}{l}\hat{\theta} \\
\dot{e} \\
0\end{array}$ & $\begin{array}{l}\hat{\theta} \\
\dot{e} \\
0\end{array}$ & $\underset{0}{\stackrel{0}{0}}$ & $\underset{0}{\stackrel{0}{0}}$ & $\underset{0}{\stackrel{0}{e}}$ & $\begin{array}{l}\hat{\theta} \\
\stackrel{e}{0} \\
0\end{array}$ \\
\hline & & 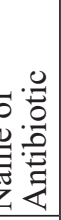 & 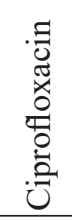 & 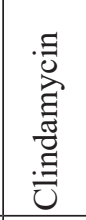 & 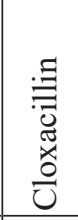 & 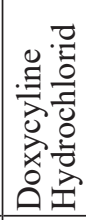 & 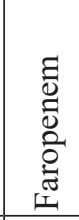 & 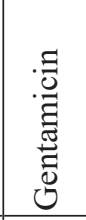 & 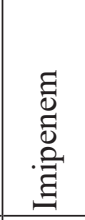 & 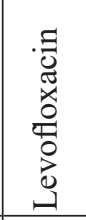 & 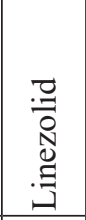 & 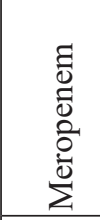 & 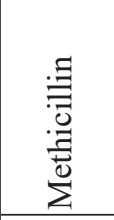 & 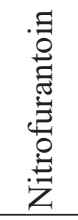 & 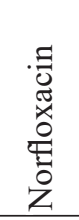 & 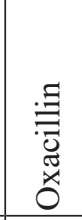 \\
\hline & & $\dot{0} \dot{z}$ & $\dot{\imath}$ & $\dot{\vec{\lambda}}$ & הี & $\dot{\lambda}$ & $\stackrel{d}{d}$ & $\ddot{v}$ & $\dot{\sim}$ & $\dot{\lambda}$ & $\stackrel{\infty}{\sim}$ & $\stackrel{\mathrm{\lambda}}{ }$ & $\dot{m}$ & $\dot{m}$ & लें & $\dot{m}$ \\
\hline
\end{tabular}




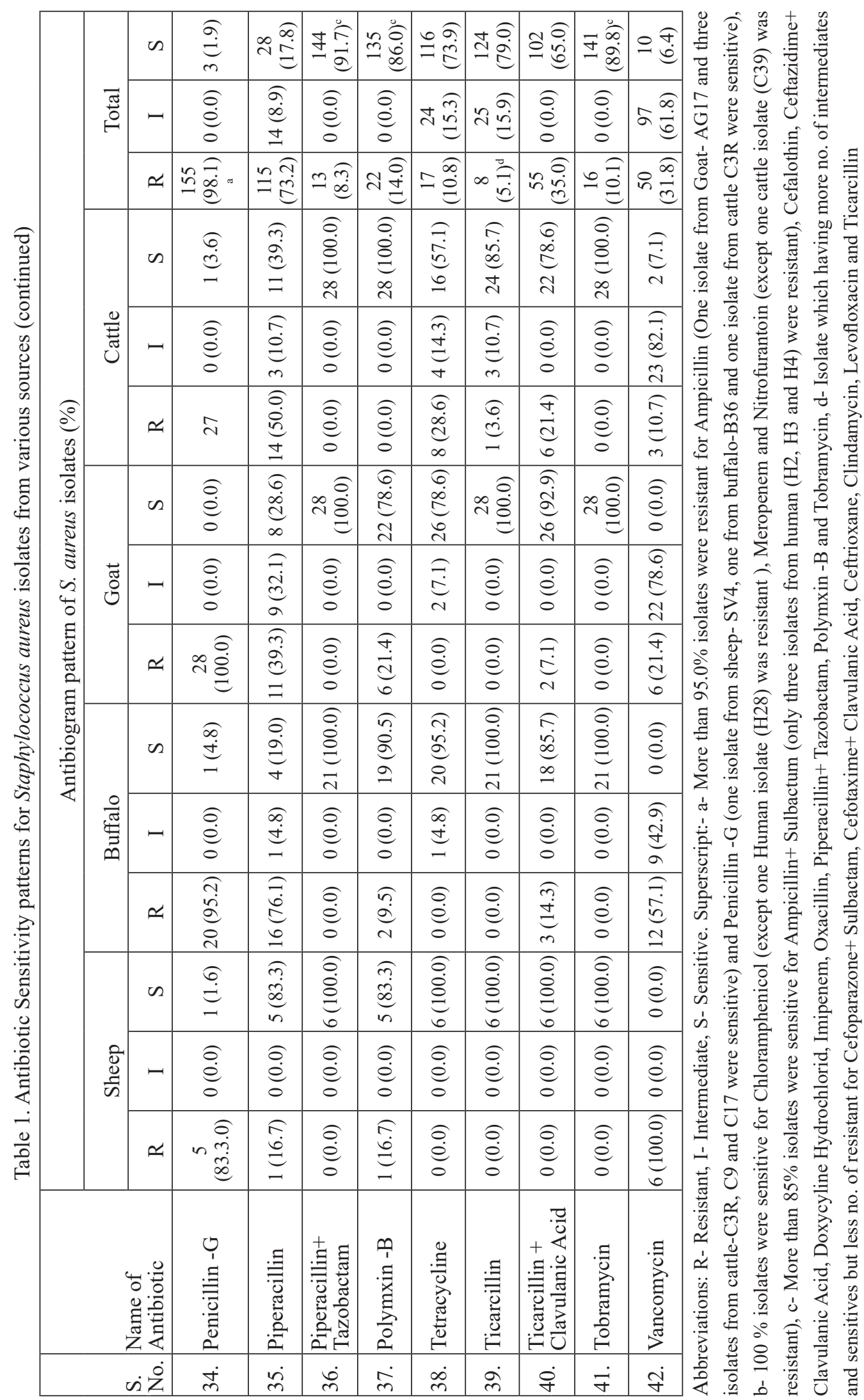


S. K. Sharma et al.: Detection and analysis of antibiotic resistance variability among Staphylococcus aureus isolates from animal and human sources

Table. 2. Analysis of variance of diameter of inhibition zone ( $\mathrm{mm}$ ) of antibiotics for $S$. aureus isolates

\begin{tabular}{|c|l|c|}
\hline S. No. & Source of variation/Antibiotic & MSS \\
\hline 1. & Ampicillin & $288.043^{* *}$ \\
\hline 2. & Ampicillin+ Sulbactum & $271.987^{* *}$ \\
\hline 3. & Azithromycin & $789.142^{* *}$ \\
\hline 4. & Aztreonam & $157.118^{* *}$ \\
\hline 5. & Cefaclor & $429.189^{* *}$ \\
\hline 6. & Cefalothin & $353.234^{* *}$ \\
\hline 7. & Cefepime & $180.461^{* *}$ \\
\hline 8. & Cefixime & $411.040^{* *}$ \\
\hline 9. & Cefixime+ Clavulanic Acid & $298.578^{* *}$ \\
\hline 10. & Cefoparazone & $152.219^{* *}$ \\
\hline 11. & Cefoparazone+ Sulbactam & $165.105^{* *}$ \\
\hline 12. & Cefotaxime & $268.889^{* *}$ \\
\hline 13. & Cefotaxime+ Clavulanic Acid & $207.341^{* *}$ \\
\hline 14. & Cefoxitin & $304.429^{* *}$ \\
\hline 15. & Ceftazidime & $134.165^{* *}$ \\
\hline 16. & Ceftazidime+ Clavulanic Acid & $163.499^{* *}$ \\
\hline 17. & Ceftrioxane & $302.930^{* *}$ \\
\hline 18. & Cefzolin & $349.944^{* *}$ \\
\hline 19. & Chloramphenicol & $25.004^{* *}$ \\
\hline 20. & Ciprofloxacin & $64.327^{* *}$ \\
\hline 21. & Clindamycin & $9.628^{\mathrm{NS}}$ \\
\hline
\end{tabular}

\begin{tabular}{|c|l|c|}
\hline S. No. & Source of variation/Antibiotic & MSS \\
\hline 22. & Cloxacillin & $476.205^{* *}$ \\
\hline 23. & Doxycycline Hydrochloride & $141.91^{* *}$ \\
\hline 24. & Faropenem & $536.097^{* *}$ \\
\hline 25. & Gentamicin & $50.272^{* *}$ \\
\hline 26. & Imipenem & $937.524^{* *}$ \\
\hline 27. & Levofloxacin & $17.739^{*}$ \\
\hline 28. & Linezolid & $41.257^{* *}$ \\
\hline 29. & Meropenem & $121.492^{* *}$ \\
\hline 30. & Methicillin & $160.922^{* *}$ \\
\hline 31. & Nitrofurantoin & $11.777^{*}$ \\
\hline 32. & Norfloxacin & $194.456^{* *}$ \\
\hline 33. & Oxacillin & $351.371^{* *}$ \\
\hline 34. & Penicillin -G & $256.907^{* *}$ \\
\hline 35. & Piperacillin & $399.747^{* *}$ \\
\hline 36. & Piperacillin+ Tazobactam & $149.886^{* *}$ \\
\hline 37. & Polymxin -B & $24.501^{* *}$ \\
\hline 38. & Tetracycline & $197.135^{* *}$ \\
\hline 39. & Ticarcillin & $322.626^{* *}$ \\
\hline 40. & Ticarcillin+ Clavulanic Acid & $350.513^{* *}$ \\
\hline 41. & Tobramycin & $77.792^{* *}$ \\
\hline 42. & Vancomycin & $10.179^{* *}$ \\
\hline
\end{tabular}

i. - Degree of freedom $(d f)=9$; ii. - * = Significant $(\mathrm{P} \leq 0.05)$; iii. - ** Highly significant $(\mathrm{P} \leq 0.01)$; iv. - NS $=$ Non-significant $(\mathrm{P}>0.05) ;$ MSS $=$ Mean Sum of Square;

Table 3. Detection of group Multiple Antibiotic Resistance Index (MAR) value among sources of S. aureus isolate

\begin{tabular}{|c|c|c|c|c|c|}
\hline $\begin{array}{l}\text { S. } \\
\text { No. }\end{array}$ & $\begin{array}{l}\text { Source of } \\
\text { Isolate }\end{array}$ & $\begin{array}{l}\text { Total No. } \\
\text { of isolate }\end{array}$ & $\begin{array}{l}\text { Aggregate antibiotic } \\
\text { resistance score }\end{array}$ & $\begin{array}{c}\text { Group MAR } \\
\text { index value }\end{array}$ & Significance \\
\hline 1. & Human & 35 & 585 & \begin{tabular}{|l|}
0.40 \\
\end{tabular} & \multirow{10}{*}{$\begin{array}{c}\text { Greater than } 0.2 \text { MAR Index of group indicates } \\
\text { that several antibiotics were used in that group and } \\
\text { more than } 0.2 \text { MAR group is an high risk potential } \\
\text { source of spread MDR }\end{array}$} \\
\hline 2. & Meat piece & 20 & 192 & 0.24 & \\
\hline 3. & Horse & 3 & 27 & 0.21 & \\
\hline 4. & Pig & 2 & 19 & 0.23 & \\
\hline 5. & Camel & 8 & 84 & 0.25 & \\
\hline 6. & Dog & 6 & 23 & 0.10 & \\
\hline 7. & Sheep & 6 & 19 & 0.08 & \\
\hline 8. & Buffalo & 21 & 89 & 0.10 & \\
\hline 9. & Goat & 28 & 100 & 0.09 & \\
\hline 10. & Cattle & 28 & 187 & 0.16 & \\
\hline
\end{tabular}

Decreasing Order of MAR index Value: - Human $>$ Camel $>$ Meat pieces $>$ Pig $>$ Horse $>$ Cattle $>$ Dog $=$ Buffalo $>$ Goat $>$ Sheep 
S. K. Sharma et al.: Detection and analysis of antibiotic resistance variability among Staphylococcus aureus isolates from animal and human sources

Table 4. Detection and Distribution of Multiple Antibiotic Resistance Index (MAR) value among individual $S$.

aureus isolates

\begin{tabular}{|c|c|c|c|c|c|c|}
\hline $\begin{array}{l}\text { S. } \\
\text { No. }\end{array}$ & $\begin{array}{l}\text { MAR Index } \\
\text { Value Type } \\
\text { (MAR) }\end{array}$ & Isolate I.D. & $\begin{array}{l}\text { No. of } \\
\text { Isolate }\end{array}$ & $\begin{array}{l}\text { No. of antibiotic, } \\
\text { which the isolate was } \\
\text { resistant }\end{array}$ & $\begin{array}{l}\text { MAR } \\
\text { Index } \\
\text { Value }\end{array}$ & Significance \\
\hline 1. & MAR1 & $\mathrm{H} 2$ & 1 & 34 & 0.81 & \multirow{21}{*}{$\begin{array}{l}66(42 \%) \text { isolates had } 0.2 \text { or } \\
\text { more than } 0.2 \text { MAR index } \\
\text { value with high risk potential } \\
\text { source of spread MDR }\end{array}$} \\
\hline 2. & MAR2 & $\mathrm{H} 1$ and $\mathrm{H} 8$ & 2 & 32 & 0.76 & \\
\hline 3. & MAR3 & $\mathrm{H} 3$ and $\mathrm{H} 4$ & 2 & 31 & 0.74 & \\
\hline 4. & MAR4 & H5 & 1 & 29 & 0.69 & \\
\hline 5. & MAR5 & $\mathrm{H} 29$ & 1 & 28 & 0.67 & \\
\hline 6. & MAR6 & H6 & 1 & 25 & 0.6 & \\
\hline 7. & MAR7 & $\mathrm{H} 27$ & 1 & 23 & 0.55 & \\
\hline 8. & MAR8 & $\mathrm{J} 4$ & 1 & 21 & 0.50 & \\
\hline 9. & MAR9 & $\mathrm{H} 25, \mathrm{Mt} 26$ & 2 & 20 & 0.48 & \\
\hline 10 & MAR10 & $\mathrm{H} 7$ and $\mathrm{H} 48$ & 2 & 19 & 0.45 & \\
\hline 11. & MAR11 & $\mathrm{H} 24, \mathrm{H} 39, \mathrm{H} 46$ and $\mathrm{C} 29$ & 4 & 18 & 0.43 & \\
\hline 12. & MAR12 & $\mathrm{H} 40$ & 1 & 17 & 0.40 & \\
\hline 13 & MAR13 & $\mathrm{H} 30$ and $\mathrm{Mt} 2$ & 2 & 16 & 0.38 & \\
\hline 14. & MAR14 & $\mathrm{H} 28, \mathrm{~J} 14$ and $\mathrm{C} 39$ & 3 & 15 & 0.36 & \\
\hline 15. & MAR15 & $\begin{array}{c}\mathrm{H} 33, \mathrm{H} 37, \mathrm{H} 45, \mathrm{Mt} 3, \mathrm{C} 37 \\
\text { and C43 }\end{array}$ & 6 & 14 & 0.33 & \\
\hline 16. & MAR16 & $\mathrm{Mt} 4, \mathrm{Mt} 9$ and $\mathrm{J} 18$ & 3 & 13 & 0.31 & \\
\hline 17. & MAR17 & Hrs3 and C12 & 2 & 12 & 0.29 & \\
\hline 18. & MAR18 & $\begin{array}{c}\mathrm{H} 12, \mathrm{H} 21, \mathrm{H} 34, \mathrm{H} 44, \mathrm{Mt} 27, \\
\text { Pg2 and C52 }\end{array}$ & 7 & 11 & 0.26 & \\
\hline 19. & MAR19 & $\begin{array}{c}\mathrm{H} 14, \mathrm{H} 22, \mathrm{Mt19}, \mathrm{J} 3, \mathrm{~B} 24, \\
\mathrm{C} 13, \mathrm{C} 41 \text { and } \mathrm{C} 46\end{array}$ & 8 & 10 & 0.24 & \\
\hline 20. & MAR20 & $\begin{array}{c}\text { H11, H15, Mt13, C34 and } \\
\text { Hrs4 }\end{array}$ & 5 & 9 & 0.21 & \\
\hline 21. & MAR21 & $\begin{array}{l}\text { H41, Mt1, Mt10, Mt15, } \\
\text { Mt22, Mt24, Mt25, Mt28, } \\
\text { Pg4, B27 and C5R }\end{array}$ & 11 & 8 & 0.20 & \\
\hline
\end{tabular}


S. K. Sharma et al.: Detection and analysis of antibiotic resistance variability among Staphylococcus aureus isolates from animal and human sources

Table 4. Detection and Distribution of Multiple Antibiotic Resistance Index (MAR) value among individual $S$. aureus isolates (continued)

\begin{tabular}{|c|c|c|c|c|c|c|}
\hline $\begin{array}{l}\text { S. } \\
\text { No. }\end{array}$ & $\begin{array}{l}\text { MAR Index } \\
\text { Value Type } \\
\text { (MAR) } \\
\end{array}$ & Isolate I.D. & $\begin{array}{l}\text { No. of } \\
\text { Isolate }\end{array}$ & $\begin{array}{l}\text { No. of antibiotic, } \\
\text { which the isolate was } \\
\text { resistant }\end{array}$ & $\begin{array}{l}\text { MAR } \\
\text { Index } \\
\text { Value }\end{array}$ & Significance \\
\hline 22. & MAR22 & $\begin{array}{c}\text { H13, Mt14, Mt20, Hrs1, J9, } \\
\text { J10, B1, B46, B55, C } 36 \text { and } \\
\text { C40 }\end{array}$ & 11 & 7 & 0.17 & \multirow{7}{*}{$\begin{array}{l}91(58 \%) \text { isolates had less } \\
\text { than } 0.2 \text { MAR index value } \\
\text { with less risk source of MDR }\end{array}$} \\
\hline 23. & MAR23 & $\begin{array}{l}\mathrm{H} 10, \mathrm{Mt} 11, \mathrm{Mt} 12, \mathrm{~J} 15, \mathrm{AG} 8 \\
\text { and G9 }\end{array}$ & 6 & 6 & 0.14 & \\
\hline 24. & MAR24 & $\begin{array}{c}\text { H9, H16, H31, J2, D7, D9, } \\
\text { SN4, B23, B26, B30, B39, } \\
\text { AG15, G24, G29, G39, G46, } \\
\text { G49, C23 and C26 }\end{array}$ & 19 & 5 & 0.12 & \\
\hline 25. & MAR25 & $\begin{array}{l}\mathrm{Mt} 31, \mathrm{D} 4, \mathrm{~B} 21, \mathrm{~B} 28, \mathrm{~B} 31, \\
\mathrm{~B} 34, \mathrm{~B} 42, \mathrm{~B} 43, \mathrm{~B} 57, \mathrm{AG} 13, \\
\mathrm{G} 1, \mathrm{G} 7, \mathrm{G} 16 \text { and } \mathrm{G} 21\end{array}$ & 14 & 4 & 0.10 & \\
\hline 26. & MAR26 & $\begin{array}{c}\text { D6, D10, D13, SV2, SV3, } \\
\text { SN3, SN14, B29, B36, AG5, } \\
\text { AG6, AG17, G2, G11, G40, } \\
\text { G41, G55, C2R, C7, C8, } \\
\text { C11, C17, C20 and C50 }\end{array}$ & 24 & 3 & 0.07 & \\
\hline 27. & MAR27 & $\begin{array}{l}\text { SV4, B5, B10, B19, AG10, } \\
\text { G10, G35, G37, G43, G45, } \\
\text { G47, C2, C9, C15, C22 and } \\
\text { C47 }\end{array}$ & 16 & 2 & 0.05 & \\
\hline 28. & MAR28 & C3R & 1 & 1 & 0.02 & \\
\hline
\end{tabular}

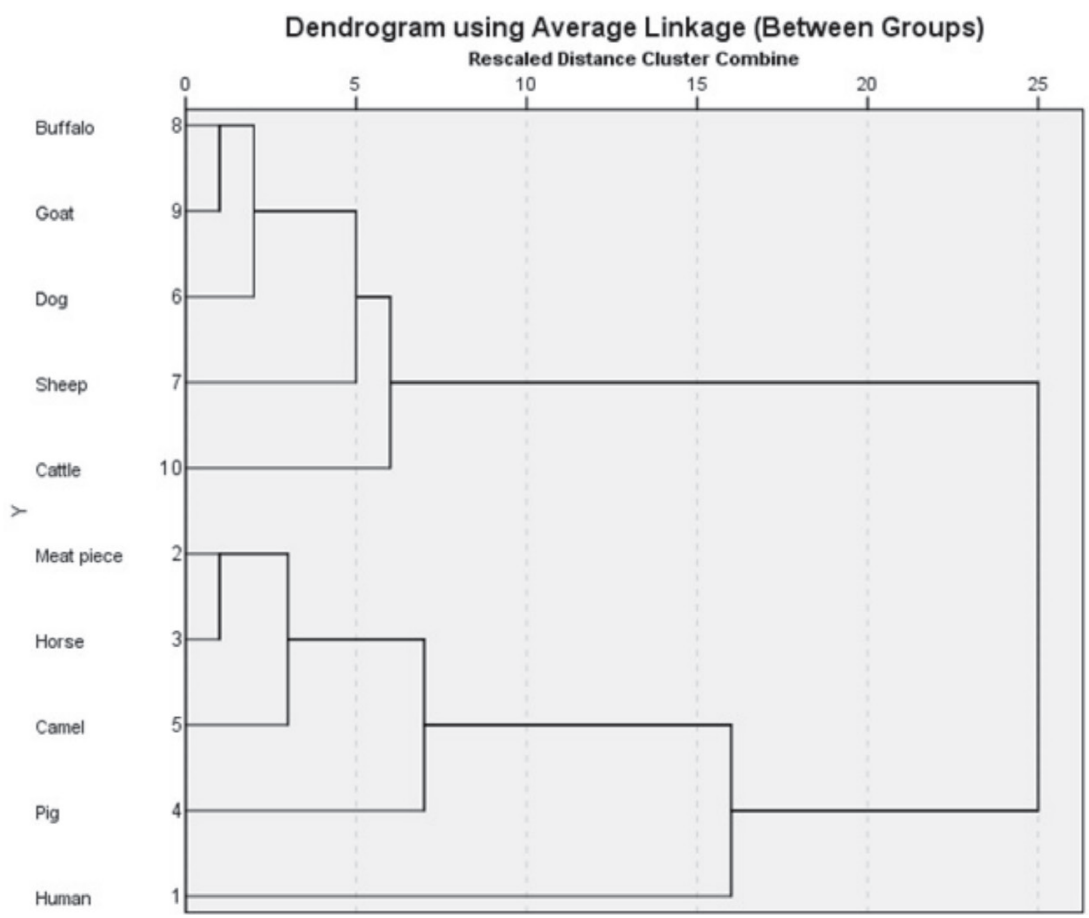

Fig. 1. Hierarchical ascendant cluster analysis of antibiotic sensitivity pattern through mean values of the diameter of the inhibition zone (mm) of antibiotics against $S$. aureus isolates 
Further, the Bonferroni correction was carried out to find the exact value of the probability level of variance among sources of $S$. aureus isolates belonging to different Duncan's Homogeneous Subsets. It was found that isolates from human sources were significantly variable, with $\mathrm{P} \leq 0.0001$ probability level of variance, in relation to other pieces of meat and animal origin sources, for most of antibiotics. Isolates from horse, camel and pig sources were non-significantly variable with each other for most antibiotics and the sheep, goat, buffalo and cattle isolates were non-significantly variable with each other for most of the antibiotics.

Hierarchical ascendant cluster analysis of antibiotic sensitivity patterns, through the mean values of the diameter of the inhibition zone $(\mathrm{mm})$ of antibiotics against $S$. aureus isolates from each group of origin, was carried out using Squared Euclidean Distance (SED) and the between-groups linkage methods (Fig. 1). The sources of isolates were clustered into three groups at 10.0 rescaled cluster distance, one cluster comprising buffalo, cattle, sheep, dog and goat sources isolates, the second cluster included horse, camel, meat piece and pig isolates, while the third cluster included human sources isolates (Fig. 1). All three clusters had significant variation $(\mathrm{P} \leq 0.05)$ between them. The first cluster of buffalo, cattle, sheep, dog and goat sources exhibited the lowest resistance and the third cluster of human isolates showed the highest resistance for most of the antibiotics (Table 1). An overall lower resistance was recorded among animal origin isolates in comparison to human origin isolates.

In the present investigation, all the $S$. aureus isolates (eExcept one cattle/C3R) were multidrug resistant (MDR) isolates. In risk assessment of MDR isolates, all multidrug resistant isolates were evaluated for both their group and individual Multiple Antibiotic Resistance (MAR) index (KRUMPERMAN, 1983). In the MAR group, $S$. aureus isolates from human sources $(0.40)$ had the highest MAR index, and sources from camels $(0.25)$, meat pieces $(0.24)$, pigs $(0.23)$ and horses (0.21) had more than 0.20 MAR in decreasing order. Other groups of $S$. aureus isolates of animal sources, such as cattle, dogs, buffalo, goats and sheep had less than 0.20 MAR, as described in Table 3. In the individual isolate MAR index, with a total of 157 isolates, 66 (42\%) isolates had a MAR index value of 0.2 or more than 0.2 , with a high risk of being a potential source of MDR spread. These isolates comprised most of the human and meat isolates, while 91 (58\%) isolates had a MAR index value less than 0.2 with less risk of being a source of MDR, and this included most of the animal origin isolates (Table 4).

\section{Discussion}

S. aureus is able to induce structural changes in the host and keeps on developing resistance against the most commonly used antibiotics. Over the last few decades, there has been a sudden increase in the use of antibiotics in veterinary as well as medical sciences, not only to control disease but also as a prophylactic measure to prevent bacterial infections secondary to viral infections. These resistant microorganisms become part of the environment and are transmitted from animals to humans, and vice versa (MEHNDIRATTA et al., 2009). Thus, the present study was designed to find variations and associations between different sources in regards to antibiotic resistance patterns, where we observed high resistance, higher MAR values and significant differences in resistance patterns between different sources.

As in the present study, we observed higher resistance for $\beta$-lactam antibiotics, GULER et al. (2005) also recorded highest resistance against $\beta$-lactam antibiotics, penicillin and ampicillin for $S$. aureus isolates from bovine clinical mastitic milk isolates. Similarly, TURUTOGLU et al. (2006), EBRAHIMI et al. (2007), PEREIRA et al. (2009) and HUSSAIN et al. (2012) also recorded maximum resistance for beta-lactam antibiotics against $S$. aureus isolates from various sources.

MOHANASOUNDARAM and LALITHA (2008) studied 150 isolates of $S$. aureus from human clinical infections, and similar results were reported for norfloxacin (100\% resistance) and chloramphenicol (18\% resistance). However, they also reported higher resistance towards tetracycline (82\%), gentamicin (88\%) and ciprofloxacin (97\%). Similar to the present study, a non-significant 
difference was observed in antibiotic sensitivity or resistance patterns against isolates from cattle and goats in the work by UPADHYAY and KATARIA (2009). Almost $80-90 \%$ of the milk isolates showed multiple drug resistance to the majority of the antimicrobial agents tested, such as: ampicillin, cloxacillin, kanamycin and vancomycin, while several isolates were found susceptible to tetracycline, oxacillin and ciprofloxacin (SHARMA et al., 2011).

YADAV et al. (2015) reported a similar antibiogram in the same area of study, who studied $32 \mathrm{~S}$. aureus isolates obtained from mastitis infections in cattle and buffalo using 33 different antibiotics. They reported doxycycline, gentamicin, methicillin and tobramycin to be more effective against all isolates, and maximum resistance was exhibited against polymxin-B and cefixime, similar to our study results. Similar to the present study, ADAMS et al. (2018) studied the epidemiological associations between $S$. aureus isolates in relation to animal breed, species of organism, sample source, and time period. They reported significant $(\mathrm{P}<0.05)$ associations between the odds of AMR and horse breed, species of organism and year. Similarly, significant $(\mathrm{P}<0.05)$ associations were identified between the odds of MDR and breed and age.

Similar to the present study ADEYEMI et al. (2015) reported more than 0.2 MAR index among $S$. aureus isolates obtained from diseased human individuals. Similarly, VIJAYALAKSHMI et al. (2013) screened $12 \mathrm{~S}$. aureus isolates from human wound samples for nine different groups of antibiotics, and found that $100 \%$ isolates were multidrug resistant with an MAR index of more than 0.22. Close to our results, UDOBI et al. (2013) reported the MAR index of $S$. aureus isolates obtained from various clinical samples from human sources, and detected that $79.6 \%, 60.6 \%$, and $76.5 \%$ of wound, skin, and bed isolates had an MAR index greater than $0.25 \%$.

SHAMILA-SYUHADA et al. (2016) studied the antibiotics resistance among $S$. aureus isolates isolated from raw milk samples obtained from small scale dairy farms, and reported MAR indexes ranging from 0.08 to 0.67 . ALI et al. (2015) studied $S$. aureus isolates from mastitic milk samples of buffalo in Egypt, and found that most of the isolates had an MAR index more than 0.28 in comparison to the present study, while only a few isolates had MAR lower than 0.2. This index is an epidemiological tool used for analysis of the risk to the environment through bacterial contamination, and it is also used to assess whether a group of isolates/an individual isolate originated from an environment where several antibiotics were used or not. The index of an isolated group of bacteria/ individual bacteria, if greater than 0.2 , implies that the strains of those bacteria originated from an environment where several antibiotics were used, and the group with MAR higher than 0.2 is an high risk potential source of spread of MDR.

Presently, there is growing concern among scientists regarding the increasing resistance in pathogens. The concerns are multifaceted, viz. inaccurate diagnosis, defective dosage, indiscriminate use, development of new drugs etc. Indiscriminate antibiotic use in dairy and other animals, as well as humans, leads to treatment failure, escalated treatment costs and development of resistance to antimicrobials. Thus, the multiplicity of the causes and emergence of resistance due to indiscriminate and prolonged use of antibiotics in the absence of an antibiogram is a major hurdle in the physical, chemical and microbiological control of infections.

In the present investigation, the highly significant difference in the antibiogram patterns between different sources of $S$. aureus may indicate the pattern and frequency of use of various antibiotics in humans and animals. The initial generations of antibiotics showed lower efficacy than the newer generations of antibiotics. The analysis of the antibiograms revealed that the susceptibility and resistance shown by the isolates was dependent on the use of the antibiotics and the source of the sample, i.e. the lower the use, the greater the susceptibility of the isolates detected. There have been many studies looking at $S$. aureus of various origins regarding their antibiogram patterns, and they have found that $S$. aureus is endowed with the capability of developing resistance towards an antibiotic, even when isolates are exposed for short periods. Further, the present study suggests that not 
only the phenotypic comparative characterization of $S$. aureus isolates from different sources but also the genotypic aspect should be explored, in relation to antibiotic resistance.

\section{Statement of animal rights}

In the present investigation no animal or human experiments, clinical trials or any invasive methods were used.

\section{Conflict of interest}

No conflict of interest exists among authors.

\section{Acknowledgements}

The authors are thankful to the Dean, College of Veterinary and Animals, Rajasthan University of Veterinary and Animal Sciences, Bikaner, for the basic facilities provided to accomplish the work. The first author is highly thankful to Department of Science and Technology, New Delhi for their financial assistance in the form of the Inspire Fellowship (IF130331).

\section{References}

ADAMS, R., J. SMITH, S. LOCKE, E. PHILLIPS, E. EROL, C. CARTER, A. ODOI (2018): An epidemiologic study of antimicrobial resistance of Staphylococcus species isolated from equine samples submitted to a diagnostic laboratory. BMC. Vet. Res 14, 42.

DOI: 10.1186/s12917-018-1367-6

ADEYEMI, F. M., K. A. AKO-NAI, E. ADEJUYIGBE, B. I. EBHODAGHE, P. O. OSHO, T. T. OYENIYI, O. O. KASSIM (2015): Molecular characterization and antibiotic resistance profiles of bacterial isolates cultured from HIV seropositive patient. Arch. Clin. Microbiol. 6, 1-11.

ALI, A. O., H. H. MAHMOUD, A. SAMEH, S. ZAKY, A. ELSAYED (2015): Antibiotic resistance profile of coagulase positive Staphylococcal infection in dairy buffaloes. World's Vet. J. 5, 46-50.

BAUER, A. W., W. M. KIRBY, J. C. SHERRIS, M. TURCK (1966): Antibiotic susceptibility testing by a standardized single disc method American. J. Clin. Pathol. 45, 493-496.

CAMPBELL, M. J., D. MACHIN, S. J. WALTERS (2007): Medical Statistics, A Text Book for the Health Sciences $4^{\text {th }}$ edn Chichester, John Wiley \& Sons Ltd.

CARTER, M. E., G. R. CARTER, J. R. COLE, JR., M. M. CHENGAPPA (1990): Staphylococcus. In: Diagnostic Procedures in Veterinary Bacteriology and Mycology. $5^{\text {th }}$ ed., Academic Press.

CLSI (2016): Performance standards for antimicrobial susceptibility testing; Twenty-sixth informational supplement CLSI document M100-S26 Wayne PA, Clinical and Laboratory Standards Institute.

EBRAHIMI. A., K. H. P. KHIRABADI, F. NIKOOKHAK (2007): Antimicrobials susceptibility of environmental bovine mastitis pathogen in west central Iran. Pak. J. Biol. Sci 10, 3014-3016.

DOI: $10.3923 /$ pjbs.2007.3014.3016

GULER, L., U. OK, K. GUNDUZ, Y. GULCU, H. H. HADIMLI (2005): Antimicrobial susceptibility and coagulase gene typing of Staphylococcus aureus isolated from bovine clinical mastitis cases in Turkey. J. Dairy. Sci. $88,3149-3154$.

DOI: $10.3168 /$ jds.S0022-0302(05)72998-2

HUSSAIN, A., S. SHAKOOR, A. YOUSAF, S. U. REHMAN, M. A. ZAMAN (2012): Clinical and subclinical Staphylococcus aureus in dairy buffaloes, disease characteristics and antibiotic susceptibility profiles of isolates. J. Anim. Plant. Sci 2, 804-811.

DOI: 10.3923/ijar. 2007.804.811

KRUMPERMAN, P. H. (1983): Multiple antibiotic resistance indexing of Escherichia coli to identify high-risk sources of fecal contamination of foods. Appl. Environ. Microbiol. 46,165-170.

LOOMBA, P. S., J. TANEJA, B. MISHRA (2010): Methicillin and vancomycin resistant $S$. aureus in hospitalized patients. J. Global. Infec. Dis. 2, 275-283.

DOI: 10.4103/0974-777X.68535

LOWY, F. D. (2003): Antimicrobial resistance, the example of Staphylococcus aureus. J. Clin. Investigation 111, $1265-$ 1273.

DOI: $10.1172 /$ JCI18535

MEHNDIRATTA, P. L., P. BHALLA, A. AHMED, Y. D. SHARMA (2009): Molecular typing of methicilineresistance Staphylococcus aureus by PCR-RFLP of spa gene, a reference laboratory prospective. Indian. J. Vet. Microbiol. 27, 116-122.

DOI: $10.4103 / 0255-0857.45363$

MOHANASOUNDARAM, K. M., M. K. LALITHA (2008): Comparison of phenotypic versus genotypic methods in the detection of methicillin resistance in Staphylococcus aureus. Indian. J. Med. Res. 127,78-84.

NABER, C. K. (2009): Staphylococcus aureus bacteremia, epidemiology pathophysiology and management strategies. Clin. Infect. Dis. 48, 231-237.

DOI: $10.1086 / 598189$.

PANTOSTI, A., M. VENDITTI (2009): Series "MRSA and the Pulmonologist”. Euro. Resp. J. 34, 1190-1196.

DOI: 10.1183/09031936.00007709

PANTOSTI, A., A. SANCHINI, M. MONACO (2007): Mechanisms of antibiotic resistance in Staphylococcus aureus. Future. Microbiol. 2, 323-334.

DOI: $10.2217 / 17460913.2 .3 .323$

PEREIRA, V., C. LOPES, A. CASTRO, J. SILVA, P. GIBBS, P. TEIXEIRA (2009): Characterization for enterotoxin production virulence factors and antibiotic susceptibility 
S. K. Sharma et al.: Detection and analysis of antibiotic resistance variability among Staphylococcus aureus isolates from animal and human sources

of Staphylococcus aureus isolates from various foods in Portugal. Food Microbiol. 26, 278-282.

DOI: $10.1016 /$ j.fm.2008.12.008

SHAMILA-SYUHADA, A. K., G. RUSUL, W. A. WANNADIAH, L. O. CHUAH (2016): Prevalence and antibiotics resistance of Staphylococcus aureus isolates isolated from raw milk obtained from small-scale dairy farms in Penang Malaysia. Pak. Vet. J. 36, 98-102.

SHARMA, D., P. K. SHARMA, A. MALIK (2011): Prevalence and antimicrobial susceptibility of drug resistant Staphylococcus aureus in raw milk of dairy cattle. Int. Res. J. Microbiol. 2, 466-470.

TURUTOGLU, H., S. ERCELIK, D. OZTURK (2006): Antibiotic resistance of Staphylococcus aureus and coagulase-negative staphylococci isolated from bovine mastitis. Bull. Vet. Ins. Pulawy 50, 41-45.
UDOBI, C. E., A. F. OBAJULUWA, J. A. ONAOLAPO (2013): Prevalence and antibiotic resistance pattern of methicillinresistant Staphylococcus aureus from an orthopaedic hospital in Nigeria. BioMed. Res. Int. 2013, 1-4.

DOI: $10.1155 / 2013 / 860467$

UPADHYAY, A., A. K. KATARIA (2009): Antibiogram of Staphylococcus aureus obtained from clinically mastitic cattle and goats. Vet. Pract. 10, 145-147.

VIJAYALAKSHMI, P., S. LALITHA, D. JEGADEESHKUMAR, P. RAJESWARI (2013): Biotyping of coagulase negative and biofilm producing wound isolates of $S$. aureus. Int. J. Sci. Innov. Discoveries 3, 376-385.

YADAV, R., S. K. SHARMA, J. YADAV, S. CHOUDHARY, A. K. KATARIA (2015): Profiling of antibiotic resistance of Staphylococcus aureus obtained from mastitic milk of cattle and buffalo. J. Pure. Appl. Microbiol. 9, 1539-1544.

Received: 1 December 2018

Accepted: 30 June 2020

\section{SHARMA, S. K., R. YADAV, S. C. MEHTA, A. K. KATARIA: Otkrivanje i analiza varijacija u otpornosti na antibiotike među izolatima bakterije Staphylococcus aureus životinja i ljudi. Vet. arhiv 90, 493-508, 2020.}

\section{SAŽETAK}

S obzirom na važnost bakterije Staphylococcus aureus u smislu otpornosti na antibiotike, cilj je ovog istraživanja bio otkriti varijacije među njezinim izolatima s obzirom na rezistenciju prema brojnim lijekovima. Istraživanje je obuhvatilo ukupno 157 vrsno specifičnih 23S rRNA S. aureus izolata iz različitih kliničkih i nekliničkih izvora životinja (goveda, bivoli, koze, ovce, psi, deve, svinje i konji), ljudi i mesa iz mesnica. Zabilježeno je više od $95 \%$ izolata otpornih na ampicilin i penicilin-G dok je gotovo $100 \%$ izolata bilo osjetljivo na kloramfenikol, meropenem i nitrofurantoin. Izolati iz različitih izvora pokazali su znakovite varijacije $(\mathrm{P} \leq 0,01)$ u rezistenciji na 39 antibiotika, znakovite varijacije $(\mathrm{P} \leq 0,05)$ za levofloksacin i nitrofurantoin, no nije bilo znakoviith varijacija $(\mathrm{P}>0,05)$ za klindamicin. Primjenom Bonferronijeve korekcije izolati iz ljudi bili su znakovito različiti $(\mathrm{P}<0,0001)$ u odnosu na uzorke iz mesa i drugih izvora animalnog podrijetla za većinu antibiotika. Izolati podrijetlom od ljudi imali su najviši MAR indeks $(0,40)$. Uočena je vrlo znakovita razlika u antibiogramima među različitim izvorima bakterije $S$. aureus što može uputiti na način i učestalost primjene različitih antibiotika u ljudi i životinja.

Klučne riječi: Staphylococcus aureus; rezistencija na antibiotike; životinje; ljudi; DMRT analiza 